\title{
Algorithm to identify circulating tumor cell clusters using in vivo flow cytometer
}

\author{
Kai Pang*, Dan Wei*, Pengfei Hai ${ }^{\dagger}$, Zhangru Yang*, \\ Xiaofu Weng* and Xunbin Wei*, \\ *State Key Laboratory of Oncogenes and Related Genes \\ Shanghai Cancer Institute \\ Med-X Research Institute and School of Biomedical Engineering \\ Shanghai Jiao Tong University \\ 1954 Huashan Road, Shanghai 200030, P. R. China \\ ${ }^{\dagger}$ Department of Biomedical Engineering \\ Washington University in St. Louis \\ One Brookings Drive, St. Louis, Missouri 63130, USA \\ ${ }^{\star}$ Radiation Oncology Center \\ Fudan University Shanghai Cancer Center (FUSCC) \\ Shanghai 200032, P. R. China \\ \$xwei01@sjtu.edu.cn
}

Received 11 February 2018

Accepted 15 May 2018

Published 22 June 2018

\begin{abstract}
Recent studies in oncology have addressed the importance of detecting circulating tumor cell clusters because circulating tumor cell clusters might survive and metastasize more easily than single circulating tumor cells. Signals with larger peak widths detected by in vivo flow cytometer (IVFC) have been used to identify cell clusters in previous studies. However, the accuracy of this criterion might be greatly degraded by variance in blood flow and the rolling behaviors of circulating tumor cells. Here, we propose a criterion and algorithm to distinguish cell clusters from single cells. In this work, we first used area-based and volume-based models for single fluorescent cells. Simulating each model, we analyzed the corresponding morphology of IVFC signals from cell clusters. According to the Rayleigh criterion, the valley between two adjacent peak signals from two distinguishable cells should be lower than $73.5 \%$ of the peak values. A novel signal processing algorithm for IVFC was developed based on this criterion. The results showed that cell clusters can be reliably identified using our proposed algorithm. Intravital imaging was also
\end{abstract}

§Corresponding author.

This is an Open Access article published by World Scientific Publishing Company. It is distributed under the terms of the Creative Commons Attribution 4.0 (CC-BY) License. Further distribution of this work is permitted, provided the original work is properly cited. 
performed to further support our algorithm. With enhanced accuracy, IVFC is a powerful tool to study circulating cell clusters.

Keywords: In vivo flow cytometer; circulating tumor cell; cell clusters; signal processing algorithm; computer simulation; Rayleigh criterion.

\section{Introduction}

Circulating tumor cells (CTCs) have been considered an important biomarker for tumor diagnosis and prognosis. ${ }^{1,2}$ Recent studies in oncology have emphasized the importance of detecting circulating tumor cell clusters. ${ }^{3-5}$ These results indicated that circulating tumor cell clusters might survive and metastasize more easily than individual circulating tumor cells. The ability to detect circulating tumor cell clusters is necessary for studies of tumor metastasis. However, conventional in vitro detection methods are insufficient. ${ }^{6-9}$ The circulating tumor cell clusters may not be present in blood samples due to limited blood sample volumes. In addition, blood sample treatments may alter the properties of circulating cell clusters. ${ }^{10,11}$ For example, the addition of an anticoagulant may disperse the cell clusters, which may be a reason that little attention has previously been paid to circulating cell clusters.

The in vivo flow cytometer (IVFC) has been used to detect and enumerate CTCs in many reported studies. ${ }^{12-32}$ With longer detection times in vivo, a larger volume of blood is examined, and CTCs are more likely to be detected with IVFC compared to conventional in vitro detection methods. To accurately identify these cell clusters, we propose a new signal processing criterion and algorithm. Signals with larger peak widths, as detected by IVFC, have been used as a criterion to identify cell clusters in previous studies. ${ }^{3}$ However, the accuracy of this criterion might be greatly degraded by changes in blood flow and the rolling behaviors of circulating tumor cells.

Here, we propose a criterion and algorithm to distinguish cell clusters from single cells, based on computer simulation and intravital imaging. In this work, we established two models of fluorescent molecule distribution for two kinds of fluorescently labeled cells. To better analyze the signals from cell clusters, we first used area-based and volume-based models for single fluorescent cells. Using simulations of each model, we analyzed the morphology of IVFC signals from single cells and cell clusters.
According to the Rayleigh criterion, the valley between two adjacent peak signals, from two distinguishable cells, should be lower than $73.5 \%$ of the peak values, and a novel signal processing algorithm for IVFC was developed based on this criterion. Our results showed that cell clusters could be reliably identified using our proposed algorithm. The results from intravital imaging also supported the use of our method.

\section{Materials and Methods \\ 2.1. Cell preparation}

The 4T1 breast cancer cells were transfected with green fluorescent protein (GFP). They were cultured and expanded in an incubator, which maintained a temperature of $37^{\circ} \mathrm{C}$ and $5 \% \mathrm{CO}_{2}$. The cells were used after the sixth passage with stable GFP expression and then the cells were prepared for the simulation using the volume-based cell model.

\subsection{Animal preparation}

Balb/c mice ( $20 \pm 2 \mathrm{~g}, 5$ week-old) were purchased from Shanghai SLAC Laboratory Animal Co. Ltd. The animal treatment procedures were approved and monitored by the Ethical Committee of Animal Experiments in the School of Biomedical Engineering, Shanghai Jiao Tong University. The mice were anesthetized with $1 \%$ pentobarbital sodium salt $(0.01 \mathrm{~mL} / \mathrm{g}$ mouse weight) during the experiment. To remove hair on the mouse ear, depilatory cream (sensitive hair removal cream; Veet) was topically applied.

\subsection{IVFC}

To simulate the proposed cell model, IVFC was used to collect signals from GFP-labeled cells. The schematic of IVFC was shown in supplementary Fig. S1. Briefly, the mouse was anesthetized and placed on the sample stage. With a $535 \mathrm{~nm}$ LED as the light source, live images of the mouse's ear were 
captured. These images were used to guide navigation of the laser beam onto a blood vessel for detection. Typically, 50-70 $\mu \mathrm{m}$-wide blood vessels were selected as the detection site. The $488 \mathrm{~nm}$ laser beams were modulated into slit-shaped beams using cylindrical lens. The size of the laser slit was approximately $5 \times 72 \mu \mathrm{m}$ at the focal plane. Under the guidance of the ear imaging by CCD camera, the laser slit was placed across the selected blood vessel. Fluorescently labeled cells were introduced into the blood by tail vein injection. When a cell passed through the laser beam, fluorescence was monitored by the detection system. After photo-electrical conversion via a photomultiplier tube (PMT), the signals were digitized $(100 \mathrm{KHz})$ and recorded on a computer.

\subsection{Computer simulation and data processing}

Computer simulations were performed on the MATLAB (2015a, Mathworks) computing platform. The plots were generated according to the area-based cell model or volume-based cell model. Data processing was performed according to the criterion and algorithm proposed using homemade MATLAB scripts.

\subsection{Intravital imaging}

Intravital imaging was performed with a Leica DM5500 system with a $10 \times$ objective (water immersion, $\mathrm{NA}=0.6$ ). The frame rate was $30 \mathrm{fps}$.

\section{Results}

\subsection{Area-based model of single cell}

To better analyze a situation where multi-cells pass through the laser slit, models for single cells were first constructed. Two different models for single cells corresponded to different fluorescent labeling methods and fluorescent dyes.

The area-based single cell model was studied first. This model could be applied to fluorescent dyes that attached surface membrane of the cells, such as DiD. To simplify the simulation model, the laser power within the laser slit beam was considered uniform. The emitted fluorescence was proportional to the area that was irradiated by the laser beam.
In this model of peak signals, key parameters included the cell radius $(R)$, width of the excitation laser $(W)$, flow speed of the cells $(v)$ and time $(t)$. When the cell size was larger than the width of laser beam, the relationship between the parameters was as follows: $R \geq \frac{1}{2} \mathrm{~W}$. The process of the cell passing through the laser beam was divided into three phases (Fig. 1(a)). The relationship between fluorescence $(F)$ and $t$ during the whole procedure is plotted in Fig. 1(b).

For the first phase, the front of the cell reached the front of the excitation laser beam, but the front of the cell had not reached the back of the excitation laser beam. This section was described as follows: $0 \leq v t \leq W$. The fluorescence $(F)$ detected within this time was described as follows:

$$
F=k S \text {. }
$$

$k$ is the ratio between the detected fluorescence and excited area, and $S$ is the laser irradiated area. During this period, $\mathrm{S}$ could be described as $2 \pi R v t$. Based on these equations, the fluorescence intensity increased proportionally within this time as

$$
F=k \pi R v t \text {. }
$$

For the second phase, the front of the cell passed the back of the excitation laser beam, but the back of the cell had not reached the front end of the excitation laser beam. This section was described as follows: $W \leq v t \leq 2 R$. In the second section, the excitation area in the cell equaled half the difference between the two ball crowns intercepted by the entire excitation laser beam. The fluorescence $(F)$ within this time was described as

$$
F=k \pi R W \text {. }
$$

The fluorescence intensity was consistent during the second section.

For the third phase, the back of the cell passed the front of the excitation laser beam, but the back of the cell had not reached the back of the excitation laser beam. This section was described as $2 R \leq v t \leq 2 R+W$. The excitation area of the cell equaled $\pi R(2 R+W-v t)$. The fluorescence $(F)$ over time was described as follows:

$$
F=k \pi R(2 R+W-v t) .
$$

From the equation above, the fluorescence intensity decreased proportionally during this section. Figures $1(\mathrm{c})$ and $1(\mathrm{~d})$ depicts the $F-t$ relationship at different flow velocities $(v)$ and radii of cells $(R)$, respectively. 
K. Pang et al.

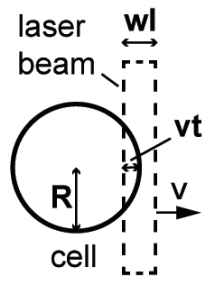

Phase one

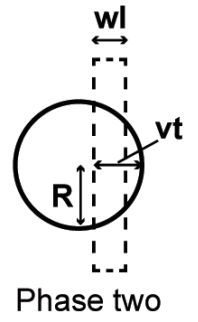

(a)

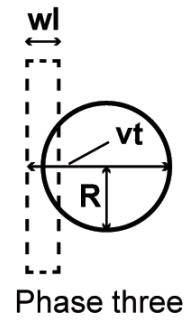

Phase three

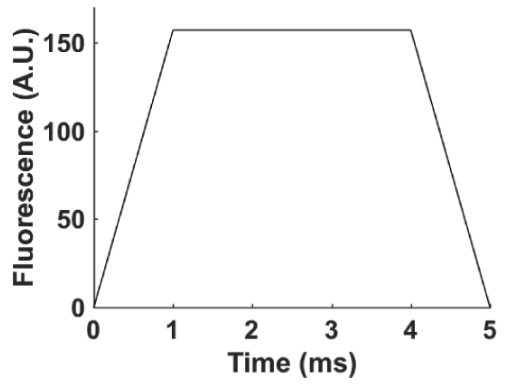

(b)

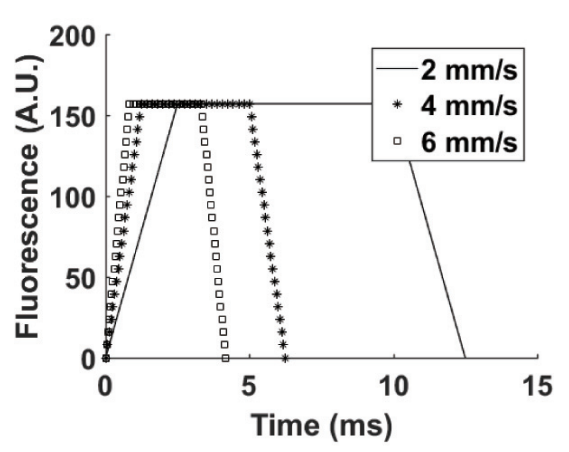

(c)

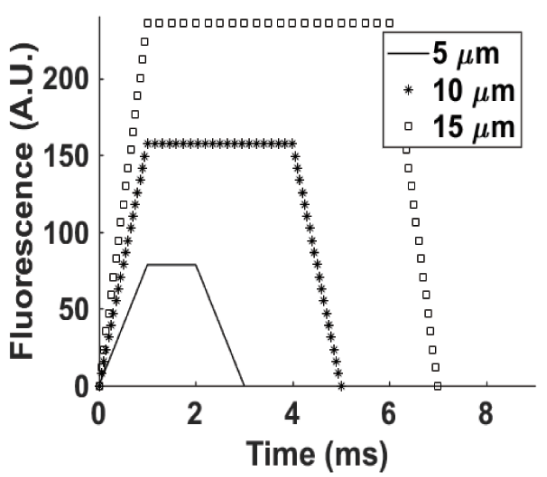

(d)

Fig. 1. Area-based single cell model. (a) Phases of cell passing the laser beam in area-based model. (b) Typical area-based model of single cell. In this model, $R=10 \mu \mathrm{m}, W=5 \mu \mathrm{m}, v=5 \mathrm{~mm} / \mathrm{s}$ and $k=1 \mathrm{mV} / \mu \mathrm{m}^{2}$. (c) Area-based model of single cell with different velocities. In this model, $R=10 \mu \mathrm{m}, W=5 \mu \mathrm{m}$ and $k=1 \mathrm{mV} / \mu \mathrm{m}^{2}$. (d) Area-based model of single cell with different radiuses. In this model, $W=5 \mu \mathrm{m}, v=5 \mathrm{~mm} / \mathrm{s}$ and $k=1 \mathrm{mV} / \mu \mathrm{m}^{2}$. A.U. denotes arbitrary unit.

\subsection{Volume-based model of single cell}

The volume-based peak model indicated that the intensity of the emitted fluorescence was proportional to volume of cells excited by the laser. In this model, fluorescence peaks corresponded to cells expressing fluorescence proteins in the cytosol, such as GFP.

In this model, key parameters included the cell radius $(R)$, width of the excitation laser $(l)$, flow speed of the cells $(v)$ and time $(t)$. When cells were larger than the excitation laser area, the relationship between the parameters was as follows: $R \geq \frac{1}{2} W$. Based on the relative positions of the cell and excitation laser beam, the cell passed through the excitation laser in three phases (Fig. 2(a)). The relationship between $F$ and $t$ during this process is plotted in Fig. 2(b).

For the first phase, the front of the cell reached the front of the excitation laser beam, but the front of the cell had not reached the back end of the excitation laser beam: $0 \leq v t \leq W$. Here, the excitation volume of the cell is the volume of the ball crown that is intercepted by the front end of the excitation laser beam. The fluorescence $(F)$ during this time is

$$
F=k V
$$

Here, $k$ is the ratio between the fluorescence intensity and excited volume, and $\mathrm{V}$ is the volume irradiated by the laser. Finally, $F$ could be described by the following equation:

$$
F=k\left(\pi R v^{2} t^{2}-\frac{1}{3} \pi v^{3} t^{3}\right) .
$$

Based on the above equation, the fluorescence intensity increased gradually during the first phase.

For the second phase, the front of the cell had passed the back of the excitation laser beam, but the back of the cell has not reached the front of the excitation laser beam. This section is described as follows: $W \leq v t \leq 2 R$. Here, the excitation volume in the cell equals the difference between the volumes of two ball crowns intercepted by the two ends of the excitation laser beam. After integrating, the 


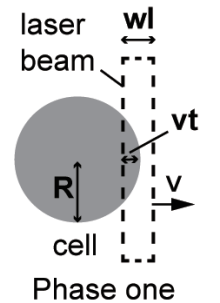

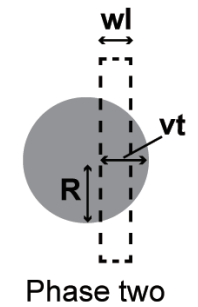

(a)
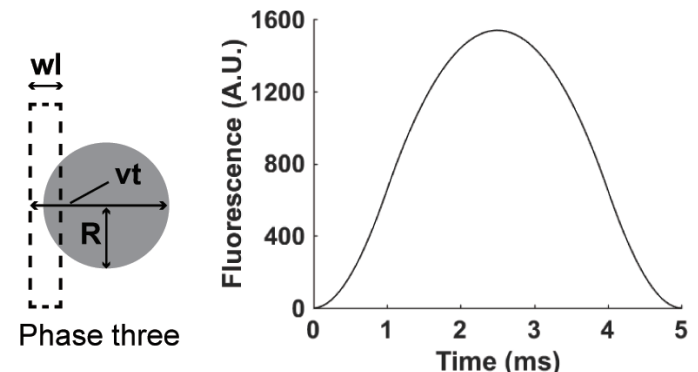

(b)

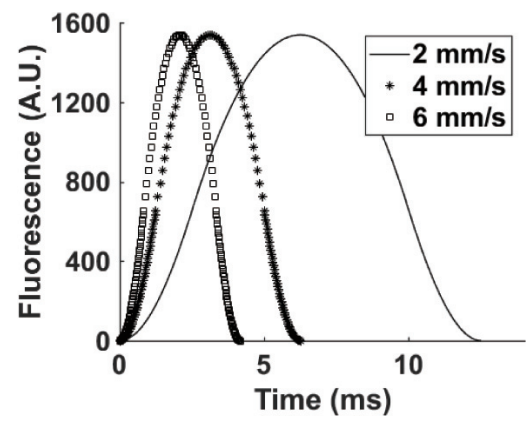

(c)

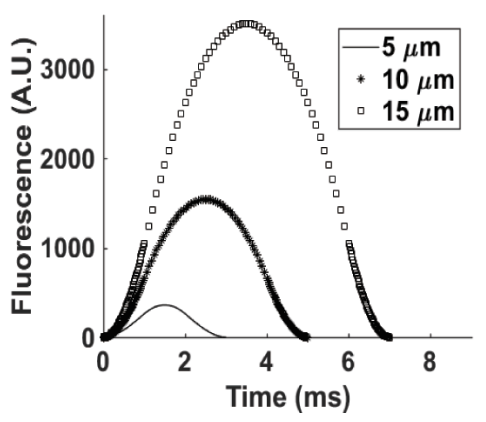

(d)

Fig. 2. Volume-based single cell model. (a) Phases of cell passing the laser beam in volume-based model. (b) Typical volume-based model of single cell. In this model, $R=10 \mu \mathrm{m}, W=5 \mu \mathrm{m}, v=5 \mathrm{~mm} / \mathrm{s}$ and $k=1 \mathrm{mV} / \mu \mathrm{m}^{2}$. (c) Volume-based model of single cell with different velocities. In this model, $R=10 \mu \mathrm{m}, W=5 \mu \mathrm{m}$ and $k=1 \mathrm{mV} / \mu \mathrm{m}^{2}$. (d) Volume-based model of a single cell with different radii of cells. In this peak model, $W=5 \mu \mathrm{m}, v=5 \mathrm{~mm} / \mathrm{s}$ and $k=1 \mathrm{mV} / \mu \mathrm{m}^{2}$. A.U. denotes arbitrary unit.

fluorescence $(F)$ within the time was as follows:

$F=k\left\{\pi R\left[v^{2} t^{2}-(v t-W)^{2}\right]-\frac{1}{3} \pi\left[v^{3} t^{3}-(v t-W)^{3}\right]\right\}$.

Based on this equation, the fluorescence intensity first increased to the local maximum and then began to fall.

For the third phase, the back of the cell has passed the front of the excitation laser beam, but the back of the cell has not reached the back of the excitation laser. This is described as follows: $v, t$ and $l: 2 R \leq v t \leq 2 R+W$. In the third phase, the excitation volume in the cell equals the volume of the ball crown intercepted by the back of the excitation laser beam. After integrating, the fluorescence $(F)$ within this time was as follows:

$$
F=k\left[\pi R(2 R+W-v t)^{2}-\frac{1}{3} \pi(2 R+W-v t)^{3}\right] .
$$

The fluorescence intensity decreased over time during the third phase. Figures 2(c) and 2(d) depict the $F-t$ relationship at different flow velocities $(v)$ and radii $(R)$ of cells, respectively.

\subsection{Models of dual-cell cluster}

To better analyze the complex cases when clusters with multiple cells pass through the excitation laser beam, models of dual-cell clusters were designed, based on the single cell models above. For the dualcell models, additional parameters were added, including the radii of the two cells $(R 1$ and $R 2)$ and the distance between the front ends of the two cells at the horizontal axis (d). The horizontal axis corresponded to blood flow in the vessels. The distances are all referred to base on this horizontal axis. If the distance between the two cells was larger than the sum of both radii and the slit, the resulting peaks would not overlap in the time domain. This condition was the same as that of two separate single peaks. The relative sizes and positions of the two cells is the key concept here.

For the first cell, the process was similar to that in the single cell model. For the second cell, the starting time changed to $t+\frac{d}{v}$, where $d$ is the 
K. Pang et al.

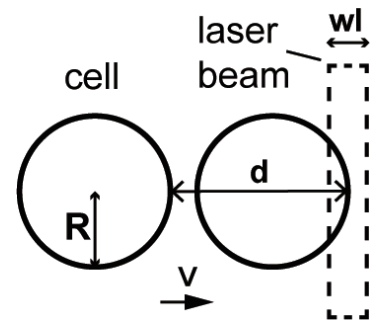

(a)
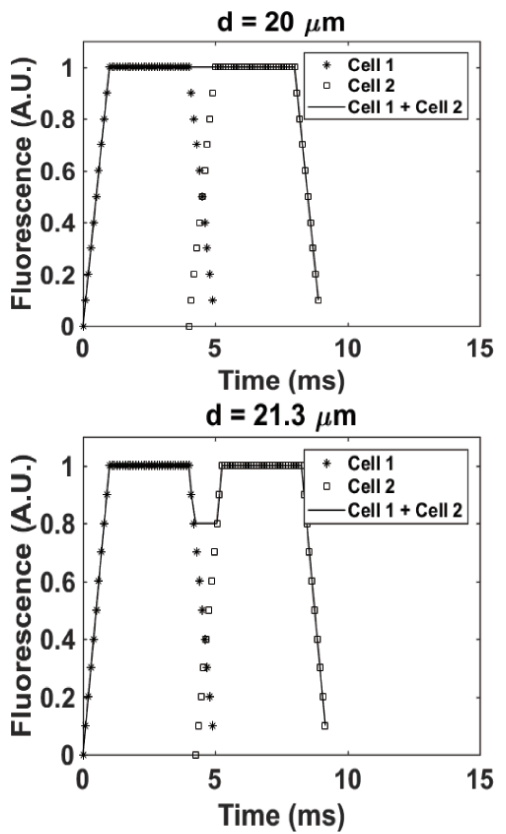

$\mathrm{d}=25 \mu \mathrm{m}$

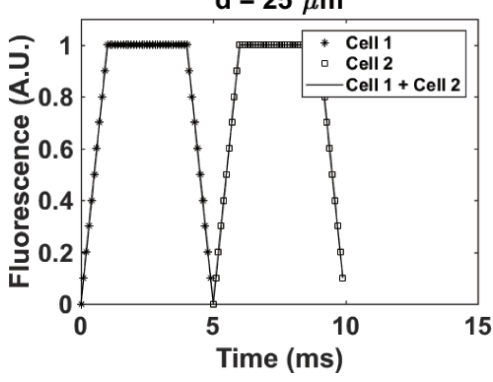

(c)

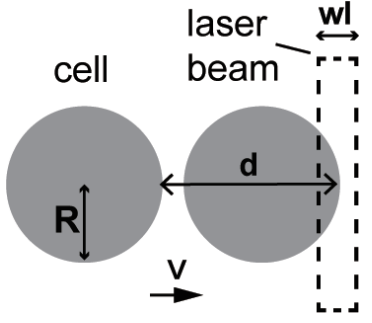

(b)
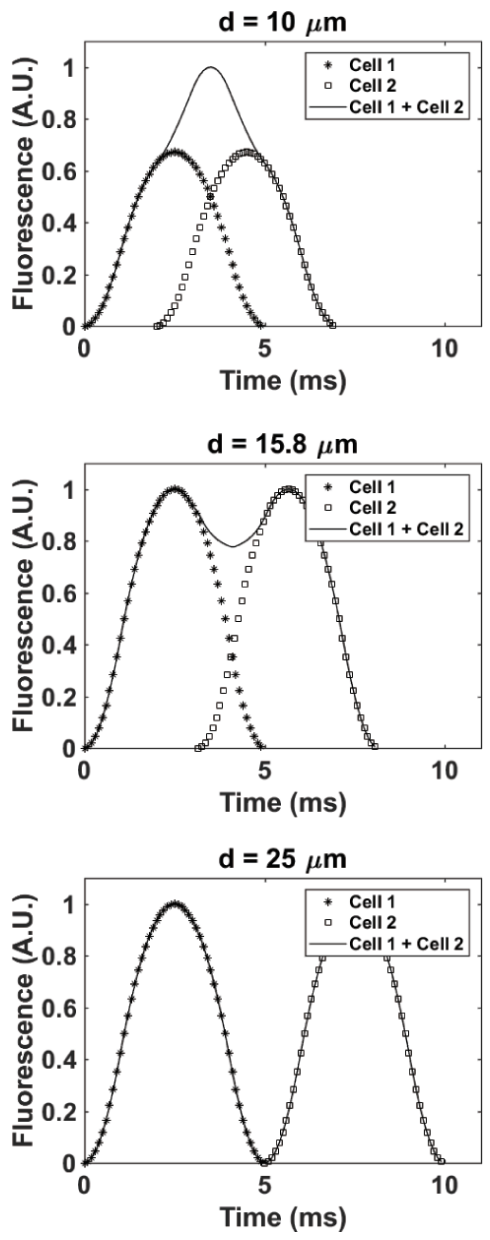

(d)

Fig. 3. Area-based and volume-based dual-cell models. (a) Phases of dual cells passing the laser beam in area-based model. (b) Phases of dual cells passing the laser beam in volume-based model. (c) Area-based dual-cell models with different cell distances. In this peak model, $v=5 \mathrm{~mm} / \mathrm{s}, k=1 \mathrm{mV} / \mu \mathrm{m}^{2}, R 1=R 2=10 \mu \mathrm{m}$ and $W=5 \mu \mathrm{m}$. (d) Volume-based dual-cell model with different cell distances. In this model, $V=5 \mathrm{~mm} / \mathrm{s}, k=1 \mathrm{mV} / \mu \mathrm{m}^{2}, R 1=R 2=10 \mu \mathrm{m}$ and $W=5 \mu \mathrm{m}$. A.U. denotes arbitrary unit.

distance between the fronts of the two cells and $v$ is the velocity of the flowing cells.

We simulated the area-based dual-cell model, and then we studied the characteristics of the dualcell model that corresponded to different distances between the two cells, as depicted in Figs. 3(a) and 3(c). To distinguish two cells in the fluorescent signals, a local minimum should exist between two adjacent local maxima, and the valley should be lower. ${ }^{33}$ Similar results could be observed using the volume-based dual-cell model, as shown in Figs. 3(b) and 3(d). 


\subsection{Rayleigh criterion and algorithm to identify cell clusters}

The resolution of in vivo flow cytometry was based on optical imaging. To distinguish two adjacent points, there should be some differences in the brightness of an overlapping area between the two diffraction spots. According to the Rayleigh criterion, ${ }^{34-36}$ the minimum value of brightness in the overlapping area should be $73.5 \%$ of the maximum value.

The algorithm to identify cell clusters was proposed based on the criterion. Briefly, the raw fluorescence data were denoised using a wavelet filter (Sym6, level 3). Then, the smoothed data trace was scanned to identify all local maxima, and a threshold was calculated according to the following equation:

$$
\begin{aligned}
\text { Threshold }= & \text { median }(\text { data })+\operatorname{mad}(\text { data }) \\
& * \text { MadFactor },
\end{aligned}
$$

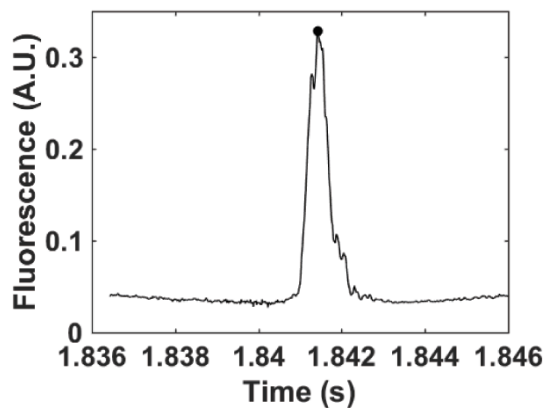

(a)

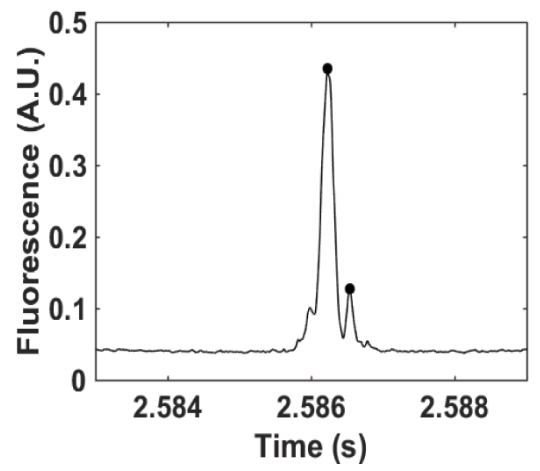

(c) where median(data) represents the median value of the data; $\operatorname{mad}($ data) indicated the absolute deviation from the mean of the data; and MadFactor was a multiplier factor to control the rigidness of the threshold, usually selected as $6 .^{37,38}$ The adjacent local maxima were clustered in a group if no local minima fell below the threshold. Effective signals from cells occurred when the local minimum between two adjacent local maxima was below $73.5 \%$ of the lower maximum.

As shown in Fig. 4(a), the effective signal for a cell was indicated by a circle. This group of signals contained only one single cell. In Fig. 4(b), there were two local maxima in this signal flock. However, the local minimum between these two local maxima was higher than $73.5 \%$ of the lower local maxima, indicated by the square. The lower peak was not regarded as an effective signal for the cell. Thus, one single cell was identified in this group of signals. A dual-cell cluster was identified, as shown in Fig. 4(c). The local minimum satisfied the Rayleigh

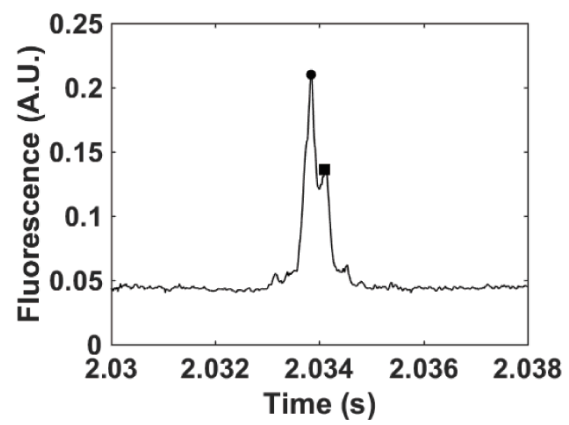

(b)

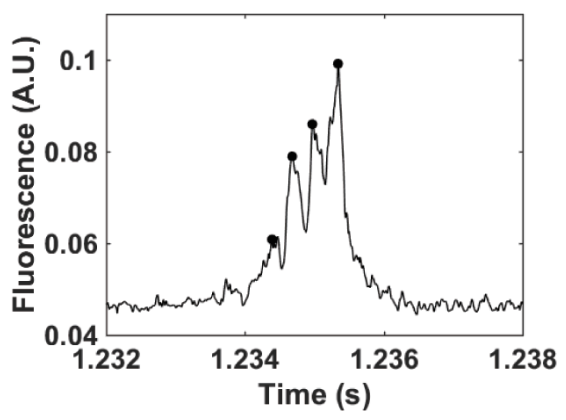

(d)

Fig. 4. Cell cluster identification using proposed algorithm based on Rayleigh criterion. (a) Typical data trace for single circulating tumor cell. Circle indicates position of local maximum. (b) Single cell signal identified by proposed algorithm. Square indicates local maximum, which is not considered an effective signal for cell. (c) Typical signal of cell cluster with dual cells. (d) Another typical signal of CTC cluster identified with proposed algorithm. This group of peaks indicates that this cell cluster consists of four cells. A.U. denotes arbitrary unit. 


\section{K. Pang et al.}

criterion. A cell luster with four cells was identified in a signal group, as depicted in Fig. 4(d). This suggested that the proposed criterion and algorithm were effective in identifying cell cluster signals.

\subsection{In vivo validation of proposed algorithm with intravital imaging}

Intravital imaging was performed to validate the proposed algorithm. The GFP-labeled 4T1 breast cancer cells were injected into the blood of a mouse via the tail vein. Fluorescence images of blood vessels on the mouse ear were continuously captured in real-time. A representative image is shown in
Fig. 5(a). Due to light absorption by hemoglobin, blood vessels appear dark in the image. The narrower vessel on the left is an artery, while the wider vessel on the right is a vein. A cell cluster consisting of two cells was observed in the vein. The part of the image within the dashed box was extracted to analyze the fluorescence profile. The fluorescence profile was calculated by integrating the fluorescence intensity within the black dotted box, which we then moved along the horizontal axis. The width of the box was $\sim 5 \mu \mathrm{m}$, which was comparable to the laser slit in IVFC. Sliding the box mimicked a cell flowing through the laser slit. After getting the whole profile, it was normalized by the second (a)

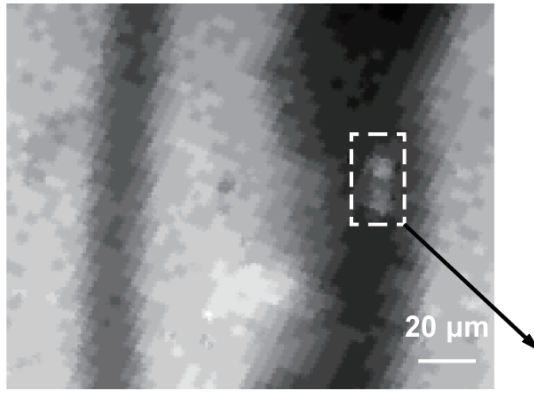

(c)

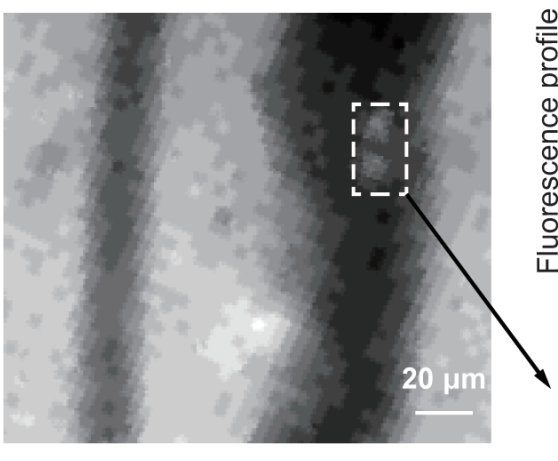

(b)

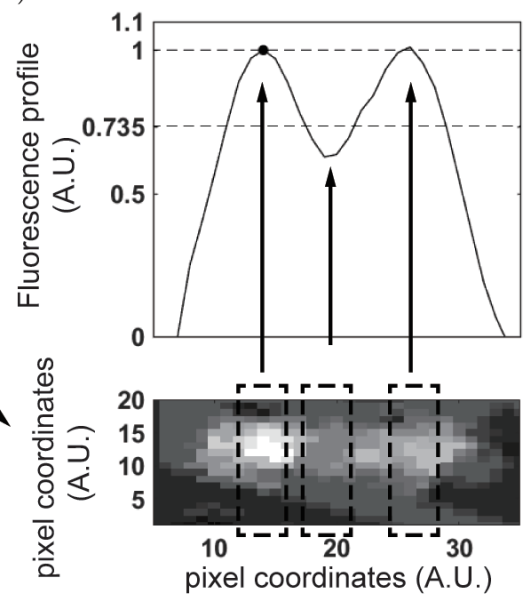

(d)

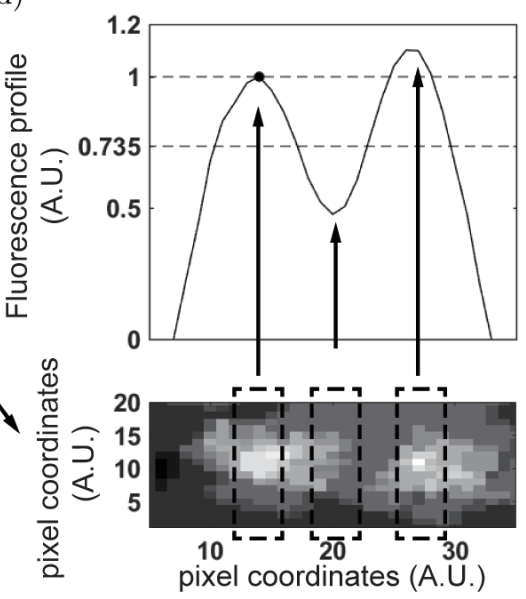

Fig. 5. In vivo validation of proposed algorithm with intravital fluorescence imaging. (a) Typical image of a $4 \mathrm{~T} 1$ cell cluster with two GFP-labeled cells. (b) Normalized fluorescence profile of the cell cluster in (a). Fluorescence profiles were calculated by integrating the fluorescence intensity within the black dotted box, which slid along the horizontal axis. Width of box was $\sim 5 \mu \mathrm{m}$, which was comparable to the laser slit in IVFC. Sliding the box mimicking the movement of a cell flowing through the laser slit. After obtaining the entire profile, it was normalized by the second largest profile value, indicated by a black circle. Normalized valley profile value between two peak profile values was below 0.735 , indicating that the flock of signal represented a dual-cell cluster and complied with the Rayleigh criterion. (c) Image of cell cluster in succeeding frame. (d) Normalized fluorescence profile of cell cluster in (c). A.U. denotes arbitrary unit. 
largest profile value indicated by a black circle. The normalized valley profile value between the two peak profile values was below 0.735 . This indicated that the group of signals represented a dual-cell cluster, complying with the Rayleigh criterion. An image of the cell cluster in the succeeding frame was shown in Fig. 5(c). Though intravital imaging was somewhat limited by motion blur, similar results were also observed. As shown in Fig. 5(d), the fluorescence profile of the cell cluster also complied with our proposed criterion.

\section{Discussion}

In this work, we established two cell models for two different fluorescently labeled cells. The area-based model corresponds to cells with fluorescent molecules distributed on the surface of cell membrane, like DiD. The volume-based model can be applied to cells producing a fluorescent protein, such as GFP. Based on these two cell models, we analyzed dualcell cluster signals, which represent the simplest type of cell clusters. Using the Rayleigh criterion, we developed a new algorithm to identify cell cluster signals. The effectiveness of the algorithm was verified with experiments.

A potential limitation of IVFC is that the collected signals provide no spatial resolution in the direction perpendicular to the blood flow. Two cells could pass through the laser slit side by side. The signals generated by these two patterns are barely distinguishable from single cell signals. In these situations, some cell clusters will be missed by IVFC. To obtain the spatial resolution, two possible solutions have been proposed and tested. One solution is to perform a line scan during data collection. The laser beam could be reshaped into a circle, and then the size of the laser beam could be reduced to generate a higher spatial resolution. A scanner could be used to move the laser spot along the direction perpendicular to the blood flow. The other solution is intravital imaging. By capturing images of flowing cells, single CTCs and cell clusters can be distinguished based on distinct morphological information. However, this solution also suffers from a limited frame rate.

\section{Conflict of Interest}

All authors declare no potential conflicts of interest.

\section{Acknowledgments}

This work was supported by a grant from the National Science Fund for Distinguished Young Scholars (Grant No. 61425006) and Program of Shanghai Technology Research Leader (Grant No. 17XD1402200).

\section{References}

1. M. Cristofanilli et al., "Circulating tumor cells, disease progression, and survival in metastatic breast cancer," N. Engl. J. Med. 351(8), 781-791 (2004).

2. J.-M. Hou et al., "Circulating tumor cells as a window on metastasis biology in lung cancer," Am. J. Pathol. 178(3), 989-996 (2011).

3. N. Aceto et al., "Circulating tumor cell clusters are oligoclonal precursors of breast cancer metastasis," Cell 158(5), 1110-1122 (2014).

4. M. Balic et al., "Circulating tumor cells: From bench to bedside," Annu. Rev. Med. 64, 31-44 (2013).

5. B. Molnar et al., "Circulating tumor cell clusters in the peripheral blood of colorectal cancer patients," Clin. Cancer Res. 7(12), 4080-4085 (2001).

6. C. Alix-Panabières, K. Pantel, "Challenges in circulating tumour cell research," Nat. Rev. Cancer 14(9), 623-631 (2014).

7. S. L. Stott et al., "Isolation of circulating tumor cells using a microvortex-generating herringbonechip," Proc. Nat. Acad. Sci. 107(43), 18392-18397 (2010).

8. A. van de Stolpe et al., "Circulating tumor cell isolation and diagnostics: Toward routine clinical use," Cancer Res. 71(18), 5955-5960 (2011).

9. M. Yu et al., "Circulating tumor cells: Approaches to isolation and characterization," J. Cell Biol. 192(3), 373-382 (2011).

10. H. W. Hou et al., "Isolation and retrieval of circulating tumor cells using centrifugal forces," Sci. Rep. 3, 1259 (2013).

11. A. F. Sarioglu et al., "A microfluidic device for labelfree, physical capture of circulating tumor cell clusters," Nat. Meth. 12(7), 685-691 (2015).

12. Y. Suo et al., "Near infrared in vivo flow cytometry for tracking fluorescent circulating cells," Cytometry A 87(9), 878-884 (2015).

13. D. A. Sipkins et al., "In vivo imaging of specialized bone marrow endothelial microdomains for tumour engraftment," Nature 435(7044), 969-973 (2005).

14. J. Novak et al., "In vivo flow cytometer for real-time detection and quantification of circulating cells," Opt. Lett. 29(1), 77-79 (2004).

15. I. Georgakoudi et al., "In vivo flow cytometry a new method for enumerating circulating cancer cells," Cancer Res. 64(15), 5044-5047 (2004). 


\section{K. Pang et al.}

16. Z. Fan et al., "In vivo tracking of'color-coded'effector, natural and induced regulatory $\mathrm{T}$ cells in the allograft response," Nat. Med. 16(6), 718 (2010).

17. Z. Fan and X. Wei, "In vivo flow cytometry: A powerful optical technology to detect circulating tumor cells and diagnose cancer metastasis in vivo/ In-vivo-Durchflusszytometrie: Ein leistungsstarkes optisches Verfahren zur Detektion zirkulierender Tumorzellen und zur In-vivo-Diagnose von Metastasen," Photonics Lasers Med. 2(1), 27-35 (2013).

18. E. I. Galanzha et al., "In vivo magnetic enrichment and multiplex photoacoustic detection of circulating tumour cells," Nat. Nanotechnol. 4(12), 855 (2009).

19. E. I. Galanzha et al., "In vivo, noninvasive, labelfree detection and eradication of circulating metastatic melanoma cells using two-color photoacoustic flow cytometry with a diode laser," Cancer Res. 69 (20), 7926-7934 (2009).

20. E. I. Galanzha et al., "In vivo acoustic and photoacoustic focusing of circulating cells," Sci. Rep. 6, 21531 (2016).

21. I. Georgakoudi et al., "In vivo flow cytometry: A new method for enumerating circulating cancer cells," Cancer Res. 64(15), 5044-5047 (2004).

22. M. V. Khodakovskaya et al., "Complex genetic, photothermal, and photoacoustic analysis of nanoparticle-plant interactions," Proc. Nat. Acad. Sci. 108(3), 1028-1033 (2011).

23. J.-W. Kim et al., "Golden carbon nanotubes as multimodal photoacoustic and photothermal high-contrast molecular agents," Nat. Nanotechnol. 4(10), 688 (2009).

24. S. Lee et al., "Real-time in vivo imaging of the beating mouse heart at microscopic resolution," Nat. Commun. 3, 1054 (2012).

25. Y. Li et al., "Circulation times of hepatocellular carcinoma cells by in vivo flow cytometry," Chin. Opt. Lett. 8(10), 953-956 (2010).

26. Y. A. Menyaev et al., "Optical clearing in photoacoustic flow cytometry," Biomed. Opt. Exp. 4(12), 3030-3041 (2013).
27. Z. A. Nima et al., "Circulating tumor cell identification by functionalized silver-gold nanorods with multicolor, super-enhanced SERS and photothermal resonances," Sci. Rep. 4, 4752 (2014).

28. J. Shao et al., "Photothermal nanodrugs: Potential of TNF-gold nanospheres for cancer theranostics," Sci. Rep. 3, 1293 (2013).

29. Y. Suo et al., "Proportion of circulating tumor cell clusters increases during cancer metastasis," Cytometry A 91(3), 250-253 (2017).

30. X. Wang et al., "Cell counting for in vivo flow cytometry signals with baseline drift," J. Innov. Opt. Health Sci. 10(03), 1750008 (2017).

31. J. Yan et al., "Circulating tumor cells are correlated with disease progression and treatment response in an orthotopic hepatocellular carcinoma model," Cytometry A 87(11), 1020-1028 (2015).

32. M.-C. Zhong et al., "Trapping red blood cells in living animals using optical tweezers," Nat. Commun. 4, 1768 (2013).

33. M. Sarimollaoglu et al., "In vivo photoacoustic timeof-flight velocity measurement of single cells and nanoparticles," Opt. Lett. 36(20), 4086-4088 (2011).

34. P. Lasch, D. Naumann, "Spatial resolution in infrared microspectroscopic imaging of tissues," Biochim. Biophys. Acta, Biomembr. 1758(7), 814-829 (2006).

35. S. Ram, E. S. Ward, R. J. Ober, "Beyond Rayleigh's criterion: A resolution measure with application to single-molecule microscopy," Proc. Nat. Acad. Sci. USA 103(12), 4457-4462 (2006).

36. F. Tamburini et al., "Overcoming the Rayleigh criterion limit with optical vortices," Phys. Rev. Lett. 97(16), 163903 (2006).

37. Y. Li et al., "Circulation times of prostate cancer and hepatocellular carcinoma cells by in vivo flow cytometry," Cytometry A 79(10), 848-854 (2011).

38. Y. Ding et al., "Signal and depth enhancement for in vivo flow cytometer measurement of ear skin by optical clearing agents," Biomed. Opt. Exp. 4(11), 2518-2526 (2013). 